\section{Up-to-date Doppler techniques for breast tumor vascularity: superb microvascular imaging and contrast-enhanced ultrasound}

\author{
Ah Young Park ${ }^{1,2}$, Bo Kyoung Seo ${ }^{1}$ \\ 'Department of Radiology, Korea University Ansan Hospital, Korea University College of \\ Medicine, Ansan; '2Department of Radiology, CHA Bundang Medical Center, CHA University, \\ Seongnam, Korea
}

Ultrasonographic Doppler techniques have improved greatly over the years, allowing more sophisticated evaluation of breast tumor vascularity. Superb microvascular imaging (SMI) and contrast-enhanced ultrasound (CEUS) with second-generation contrast agents are two representative up-to-date techniques. SMI is a sensitive Doppler technique that adopts an intelligent filter system to separate low-flow signals from artifacts. With the development of second-generation contrast agents, CEUS has also emerged as a useful Doppler technique for evaluating tumor microcirculation. Both techniques can improve the diagnostic performance of gray-scale ultrasonography by providing vascular information useful not only for the morphologic assessment of microvessels, but also for the quantitative analysis of perfusion. In this review, we explain the imaging principles and previous research underlying these two vascular techniques, and describe our clinical experiences.

Keywords: Breast; Ultrasonography, Doppler; Contrast media; Microvessels

\section{Introduction}

Tumor angiogenesis is the non-physiologic proliferation of blood vessels penetrating into cancerous tumors [1]. Tumor angiogenesis is fundamental for tumor growth, progression to invasive cancer, and metastasis $[1,2]$. Numerous anti-angiogenic agents are currently in use or under development based on our understanding of the angiogenic mechanism of breast cancer $[1,3]$. Therefore, the clinical assessment of tumor vascularity can help diagnose breast cancer, choose a management plan, and predict the prognosis. The gold standard for assessing tumor angiogenesis is immunohistochemical evaluation of microvessel density (MVD). MVD is highest in histopathologically aggressive ductal carcinoma in situ and is correlated with a greater likelihood of metastasis [4].

Color Doppler imaging (CDI) and power Doppler imaging (PDI) are widely available techniques used as adjuncts to gray-scale ultrasonography (US). Doppler signs suggestive of malignancy such as hypervascularity, central vascularity, and penetrating or branching vessels can help differentiate breast

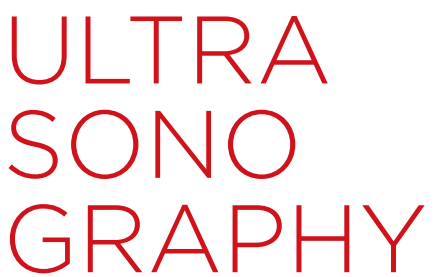

REVIEW ARTICLE

https://doi.org/10.14366/usg. 17043 pISSN: 2288-5919 - elSSN: 2288-5943 Ultrasonography 2018;37:98-106

Received: June 14, 2017

Revised: August 9, 2017

Accepted: August 19, 2017

Correspondence to: Bo Kyoung Seo, MD, Department of Radiology, Korea University Ansan Hospital, Korea University College of Medicine, 123 Jeokgeum-ro, Danwongu, Ansan 15355, Korea

Tel. +82-31-412-5229

Fax. +82-31-412-5224

E-mail: seoboky@korea.ac.kr

This is an Open Access article distributed under the terms of the Creative Commons Attribution NonCommercial License (http://creativecommons.org/ licenses/by-nc/3.0/) which permits unrestricted noncommercial use, distribution, and reproduction in any medium, provided the original work is properly cited.

Copyright @ 2018 Korean Society of Ultrasound in Medicine (KSUM)

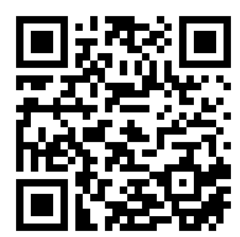

How to cite this article:

Park AY, Seo BK. Up-to-date Doppler techniques for breast tumor vascularity: superb microvascular imaging and contrast-enhanced ultrasound. Ultrasonography. 2018 Apr;37(2): 98-106. 
cancer from benign tumors [5-7]. However, there is a significant overlap of Doppler features between benign and malignant tumors because conventional Doppler imaging has limitations in detecting low-quantity and slow flow [8].

Recently, more advanced Doppler techniques and US contrast agents have been introduced to assess tumor vascularity more effectively. Superb microvascular imaging (SMI; Aplio 500 and later models from Toshiba Medical Systems Corporation, Tokyo, Japan) is a novel Doppler technique that improves sensitivity for microvessels, with high resolution and fewer motion artifacts. Contrast-enhanced ultrasound (CEUS) with second-generation contrast agents is another sensitive Doppler technique enabling the evaluation of tumor microcirculation and perfusion. In this review, we explain the imaging principles and earlier research underlying these two vascular techniques and describe our clinical experiences.

\section{Superb Microvascular Imaging}

\section{Imaging Principles of SMI}

Ultrasonic Doppler signals derive not only from blood flow, but also from tissue motion (clutter). The clutter signals overlap the lowspeed flow components. Conventional Doppler techniques apply a single-dimensional wall filter to remove clutter, resulting in loss of the slow component. In contrast, SMI uses a multidimensional filter to separate flow signals from clutter, thus removing only the clutter and preserving the slow flow signals (Fig. 1) [9, 10]. Fig. 2 shows a comparison of vascular findings among CDI, PDI, and SMI.

\section{Imaging Acquisition of SMI}

SMI provides two modes of vascular imaging: color and monochrome. The color mode demonstrates gray-scale and color information simultaneously. When using the color mode, it is possible to control the time smoothing function-also known as frame averaging-from grade 1 to 7 . Increasing the time smooth improves temporal resolution by accumulating the flow signals acquired frame-by-frame, allowing vessel continuity to be evaluated more accurately. The monochrome mode focuses only on the vasculature, with its sensitivity improved by subtracting the background information. It is displayed side-by-side with the grayscale image.

To optimize the image quality, it is important to understand the Doppler imaging parameters and to become familiar with practical tips for SMI examinations. In Doppler imaging, various parameters can influence the imaging quality, and these parameters interact in complex manners. To increase sensitivity to flow signals, color gain should be increased to make the intensity of flow more apparent on the monitor [11]. In addition, the region of interest (ROI) width should be reduced to improve sensitivity. Increasing the ROI width reduces the frame rate because multiple pulses are needed for each line of sight [11]. In examinations using an early version of SMI (ver. 5.0, Aplio 500) that did not possess a direct scale control function, the scale could be controlled by changing the ROI width. In our experience, decreasing the ROI width to a scale less than $2.5 \mathrm{~cm} /$ sec is recommended for improving the visualization of microvessels. Subsequent versions of SMI equipment provide a direct-control scale function. However, increasing color gain and decreasing the $\mathrm{ROI}$ width also lead to an increase in flash artifacts. Therefore, it is important to achieve maximal sensitivity without developing flash artifacts by upregulating color gain or downregulating ROI width in order to initially develop a flash artifact, and then to adjust these settings gradually until the flash artifact disappears. ROI depth is also associated with the frame rate, because increasing the ROI depth results in a longer wait time for returning echoes [11]. Therefore, the ROI setting should be as small and superficial as
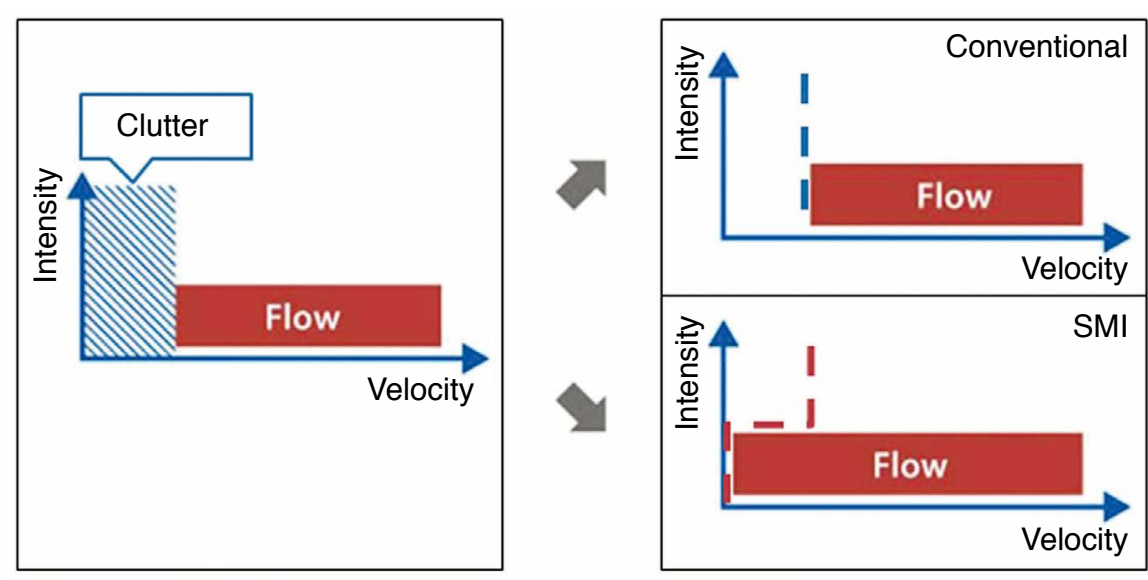

Fig. 1. Imaging principles of superb microvascular imaging (SMI). The graph in the left column shows the overlap of a tissue motion artifact (clutter) and low-speed flow signals. The top right graph shows that conventional Doppler techniques apply a single-dimensional wall filter (blue dotted line) to remove clutter, resulting in the loss of the slow flow signals. The bottom right graph shows that the SMI technique applies a multidimensional filter (red dotted line) to remove only the clutter, preserving the slow flow signals. Adapted from Hata J (2014), Seeing the unseen new techniques in vascular imaging, with permission of Toshiba Medical Systems Corporation [10]. 
possible to improve sensitivity for microvessels and image resolution. Making patients hold their breath can be helpful to reduce motion artifacts. Finally, pressing the target lesion gently with the probe prevents the collapse of microvessels.

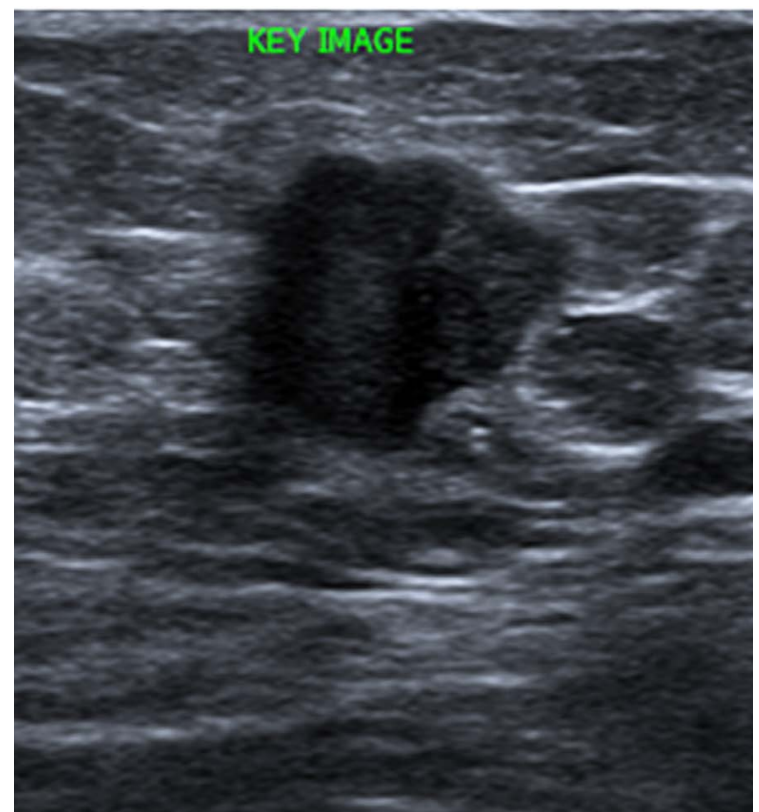

A

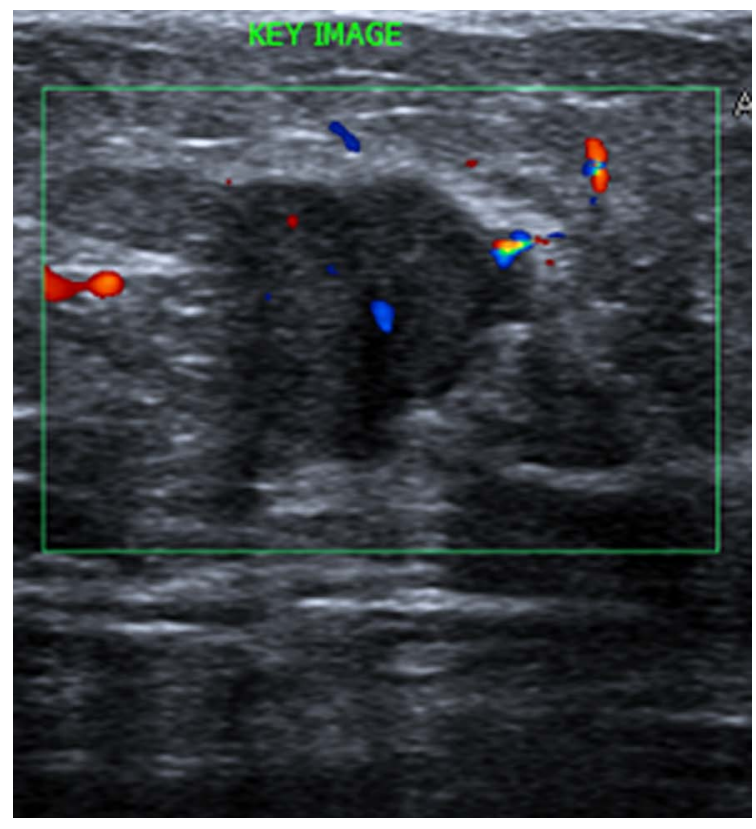

C

\section{Utility of SMI in Breast Tumor Evaluation}

Recently, several studies have reported SMI to be superior to CDI or PDI in evaluating breast tumor vascularity and improving the diagnostic performance of US [9,12-15]. Ma et al. [12] reported

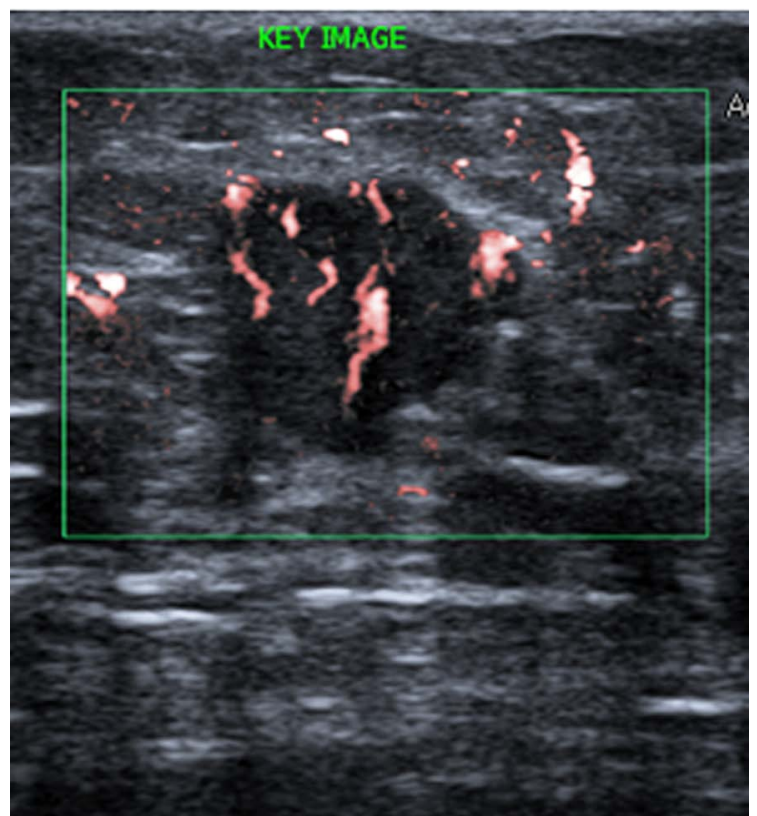

B

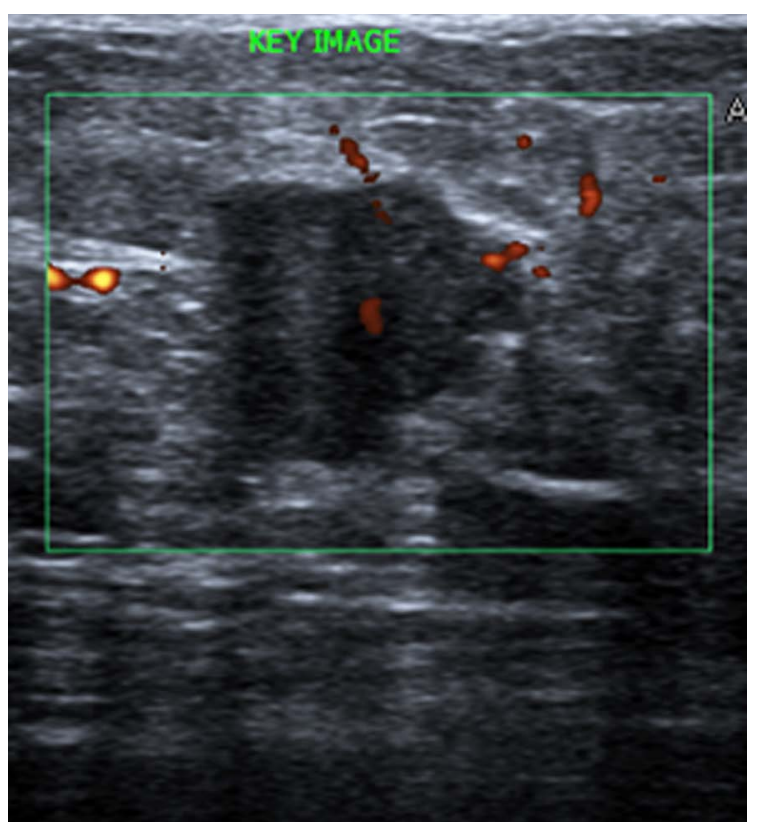

D

Fig. 2. Vascular findings of color Doppler ultrasonography, power Doppler ultrasonography, and superb microvascular imaging (SMI) of a 65-year-old woman with invasive ductal carcinoma.

A. Gray-scale ultrasonography shows an irregular indistinct or microlobulated hypoechoic mass in the left breast, assessed as, Breast Imaging Reporting and Data System category 4C. B. SMI shows multiple branching and penetrating vessels within the mass. C, D. Color Doppler ultrasonography (C) and power Doppler ultrasonography (D) show a few dot-like vessels within the mass. 
that the degree of blood flow assessed with a grading system from 0 to 3 was significantly higher on SMI than on CDI, and that the difference in the value of vessel numbers between SMI and CDI ("SMI-CDI") showed the best diagnostic performance in discriminating malignant from benign tumors (area under the curve [AUC] of "SMI-CDI" vs. AUC of SMI and CDI: 0.89 vs. 0.81 and 0.73 , respectively) [16]. Park et al. [9] reported that SMI was the best technique for detecting a larger number of cancer vessels (mean numbers of vessels, $7.2 \pm 3.0$ on SMI, $2.5 \pm 2.4$ on $\mathrm{CDI}$, and $2.8 \pm 3.0$ on PDI) and evaluating penetrating or branching vessel morphology and both peripheral and central vascular distribution. Yongfeng et al. [13] reported that SMI detected a larger number of flow signals than PDI and showed improved sensitivity ( $86 \%$ vs. $71 \%$ ) when a centrally distributed branching or diffusing morphology was used as a criterion for malignancy. Zhan et al. [14] reported that SMI showed significantly higher median numbers of penetrating vessels in avascular masses assessed as Breast Imaging Reporting and Data System category 3 or 4, and that the diagnostic performance was improved by detecting penetrating vessels (AUC increased from 0.914 to 0.947 ). Common features suggestive of malignancy in these investigations include hypervascularity, branching or penetrating vessel morphology, and the presence of central vascularity and peripheral vascularity. These vascular signs reflect the microscopic features of angiogenesis in breast cancer, including immature capillary overgrowth from the surrounding vessel toward the inside of the lesion [2]. SMI also showed a higher correlation with MVD than $C D I(r=0.82$ vs. $r=0.68)$ in recent research by $\mathrm{Ma}$ et al. [15]. Fig. 3 demonstrates the findings of SMI for a malignant tumor that was assessed as category 4A on gray-scale US.

Furthermore, the quantitative analysis of tumor vascularity recently became possible by calculating the vascular index using

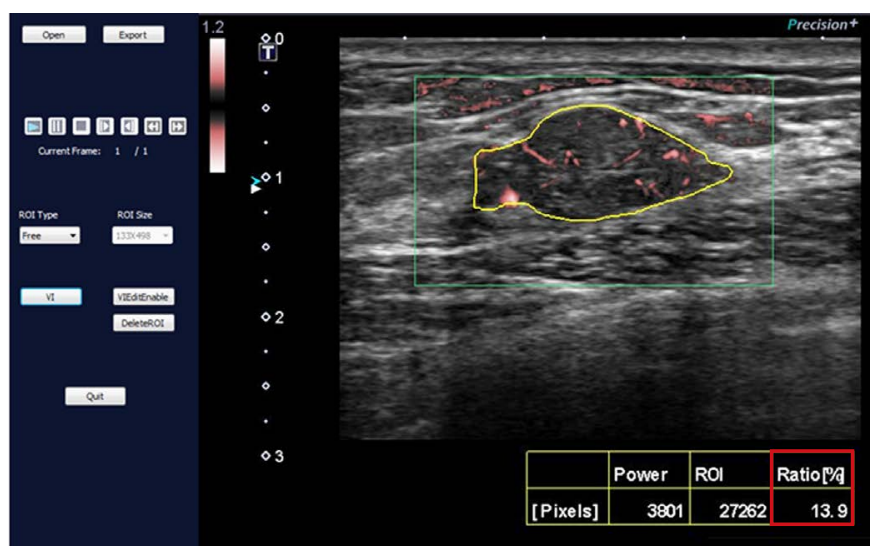

Fig. 4. Measurement of the vascular index for the superb microvascular imaging (SMI) of a 40-year-old woman with fibroadenoma. When a Doppler image or video file in Digital Imaging and Communications in Medicine format is opened in the dedicated software and the region of interest (ROI) is set as the entire target lesion (yellow line), the vascular index (red box) is automatically calculated. The vascular index is the ratio between the pixels for the Doppler signal and those for the total lesion. This SMI shows a Breast Imaging Reporting and Data System category $4 \mathrm{~A}$ mass with a few branching or penetrating vessels and a vascular index of $13.9 \%$. Biopsy revealed fibroadenoma.

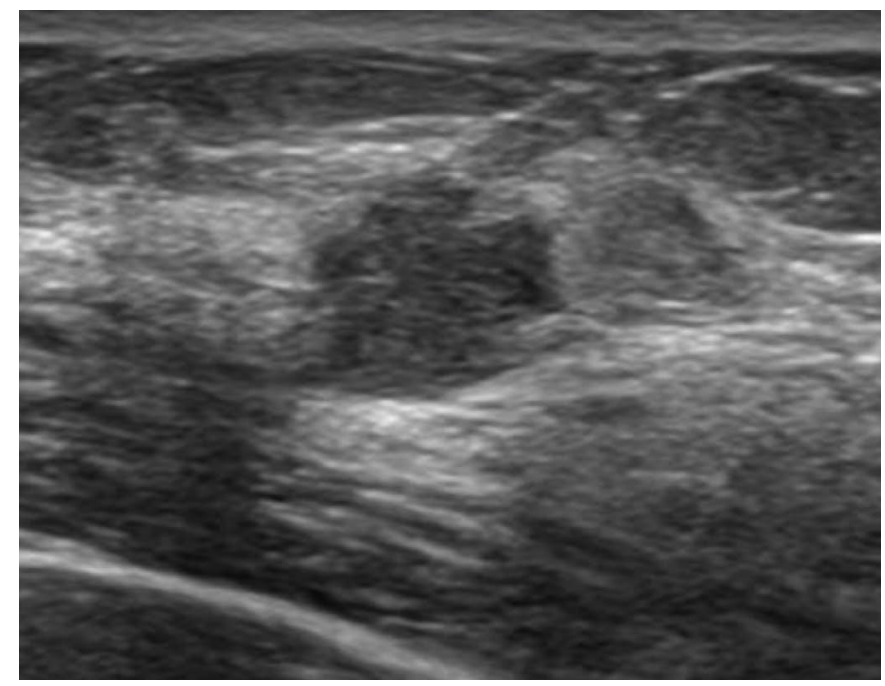

A

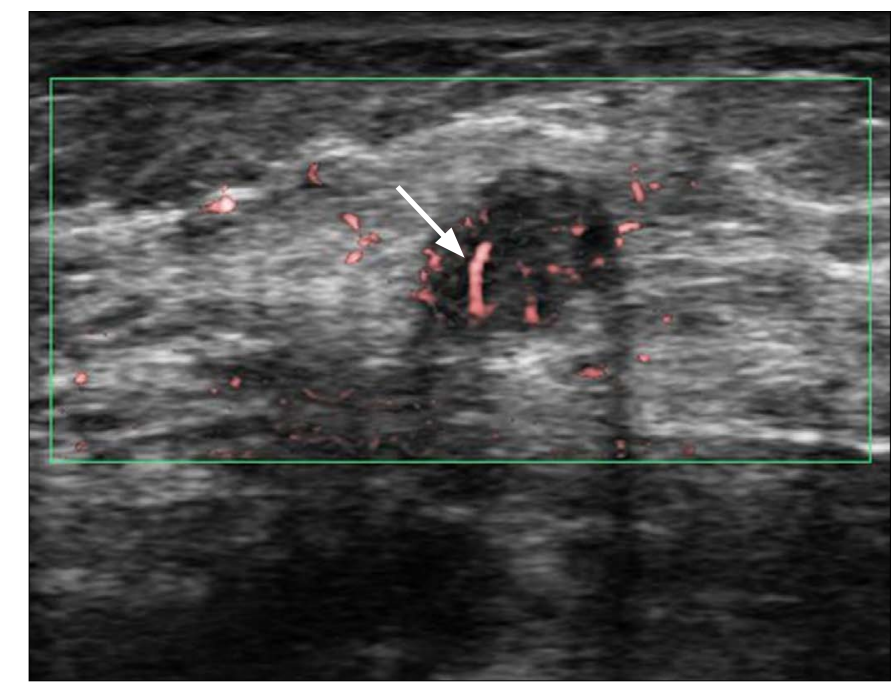

B

Fig. 3. Gray-scale ultrasonography and superb microvascular imaging (SMI) of a 53-year-old woman with invasive ductal carcinoma. A. Gray-scale ultrasonography shows an oval microlobulated hypoechoic mass in the right breast, assessed as Breast Imaging Reporting and Data System category 4A. B. SMI shows a penetrating vessel (arrow) and both peripheral and central vascularity. 
dedicated software (the VI Test App from Toshiba Medical Systems Corporation) (Fig. 4). The vascular index (\%) is the ratio between the pixels for the Doppler signal and those for the total lesion. We expect that this parameter will provide valuable information about the degree of vascularity, together with the previously discussed qualitative assessment.

\section{Potential and Limits of SMI}

SMI shows superior sensitivity for microvessels, and thus allows us to evaluate vessel complexity and distribution in more detail, which can improve the diagnostic performance for diagnosing breast malignancy. Furthermore, a quantitative analysis of the vascular index is expected to provide more objective information about the degree of vascularity. However, a measure of the learning curve is needed for SMI to be used more efficiently. Knowledge of practical tips about SMI and repeated clinical application are important for obtaining optimal-quality images.

Finally, further investigation is needed to identify the optimal cut-off value of the vascular index and qualitative criteria useful for discriminating breast cancer from benign tumors. By extension, the correlations between SMI features and various histopathologic factors, such as MVD, histologic grade, lymph node status, tumor diameter, hormone receptor status, and tumor gene expression might be one of the next areas of research to determine whether preoperative SMI examinations can be helpful in predicting the prognosis and/or choosing a treatment plan for patients with breast cancer.

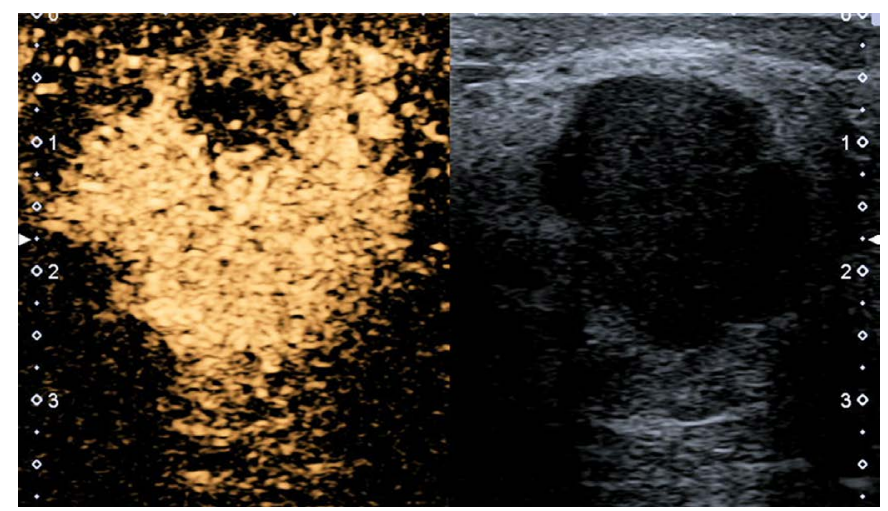

Fig. 5. Contrast-enhanced ultrasonography (CEUS) of a 52-yearold woman with invasive ductal carcinoma. During CEUS, the contrast-specific mode (left) and the gray-scale mode (right) are displayed side-by-side. The contrast agent in the vasculature enhances the backscatter of ultrasound waves and amplifies the flow signals (Aplio 500 was used as the US equipment and SonoVue as the contrast agent).

\section{Contrast-Enhanced Ultrasound}

\section{Imaging Principles of CEUS}

CEUS is the application of ultrasound contrast agents to medical US. A microbubble US contrast agent in the vasculature enhances the backscatter of US waves. This results in a marked amplification of the flow signals, providing information about the microvasculature (Fig. 5) [17].

With the introduction of second-generation US contrast agents and the development of a contrast-specific mode with a low mechanical index $\left(\mathrm{Ml}_{;}<0.3\right)$, continuous and real-time scanning has become possible $[18,19]$.

The second-generation contrast agents consist of a shell containing a polymer-based material and internal slowly diffusing gas such as hexafluoride or perfluorobutane [18]. These lowsolubility gases circulate in the blood pool with a stronger and longer-lasting signal enhancement, allowing hemodynamic evaluation. Commercially available agents include SonoVue (Bracco SpA, Milan, Italy), Definity (Lantheus Medical Imaging, North Billerica, MA, USA), and Sonazoid (Daiichi-Sankyo, Tokyo, Japan) [19]. Most investigations of breast tissue to date have used SonoVue or Sonazoid [20-28].

The $\mathrm{MI}$ is an estimate of the maximum amplitude of the pressure pulse in tissue, reflecting the power of the system [19]. A higher

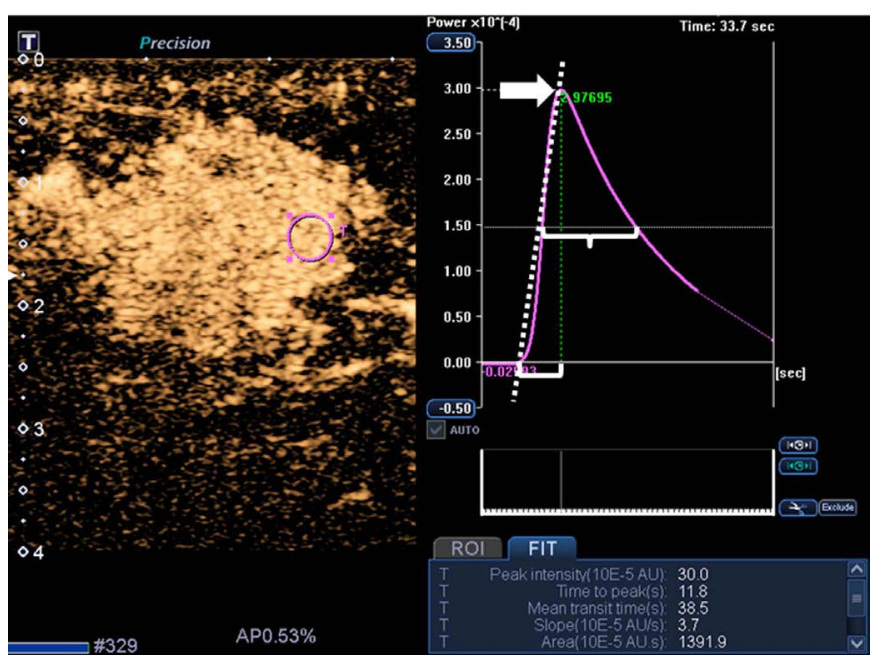

Fig. 6. Time-intensity curve analysis for a contrast-enhanced ultrasonography (CEUS) examination of a 38-year-old woman with invasive ductal carcinoma. When the region of interest (ROI) (pink circle) was set in the area of the strongest enhancement, the following quantitative parameters were automatically calculated: peak intensity (arrow), time to peak (bracket), mean transit time (brace), slope (dotted diagonal line), and area under the curve. This CEUS image was captured 34 seconds after injection of the contrast agent, when the intensity had reached its peak. 
MI corresponds to higher acoustic power, resulting in a more rapid disruption of the microbubbles [19]. Therefore, a low-MI technique enables microbubble agents to remain static.

The contrast-specific mode refers to US technology that can discriminate the non-linear signals generated by the US contrast agents from the linear signals generated by the tissue [18]. Because the non-linear signals from the tissue and the US contrast agents are proportional to the MI, a low-MI technique offers a more discriminative reception of non-linear signals from the US contrast agents by decreasing the non-US contrast agent signals [18].

\section{Imaging Acquisition of CEUS}

In CEUS examinations, the contrast-specific mode should be activated first. Immediately after the contrast agent is injected via a peripheral vein in a bolus fashion, continuous scanning for the target lesion is performed. The CEUS images can be stored as a video for repeated subsequent analyses.

With CEUS and second-generation contrast agents, both qualitative and quantitative approaches are available for image interpretation [20]. Qualitative analysis includes the evaluation of the enhancement patterns of the lesion, including the enhancement degree, order, margin, internal homogeneity, penetrating vessel, and perfusion defect. Quantitative analysis includes time-intensity curve analysis, such as the kinetic curve analysis of contrast-enhanced dynamic magnetic resonance imaging (MRI). All US systems are supplied with built-in analysis packages, and off-line software packages such as Qontrast (Bracco) are also available $[19,21]$.

In our institution, CEUS examinations were performed in two

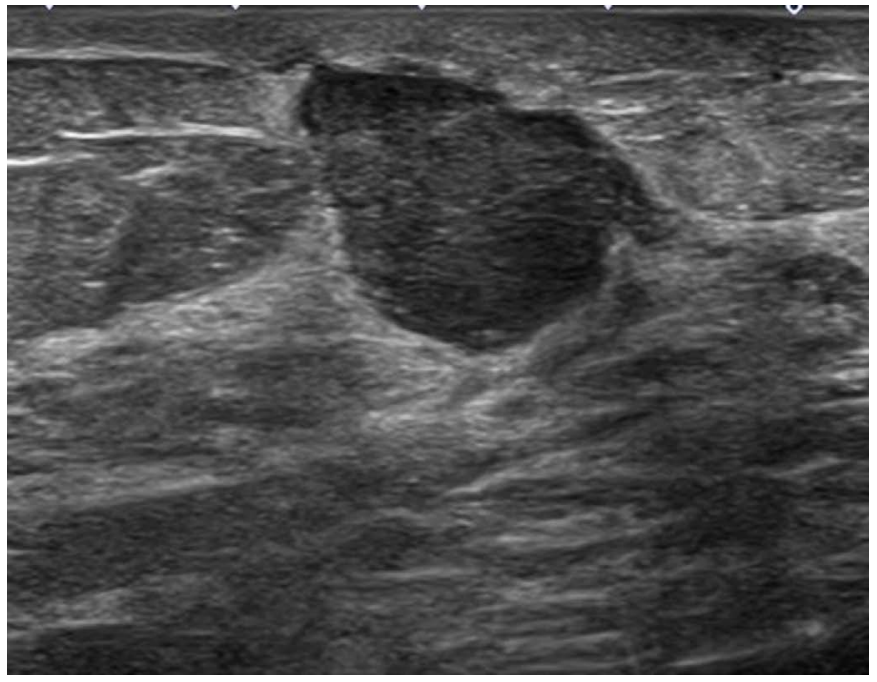

A

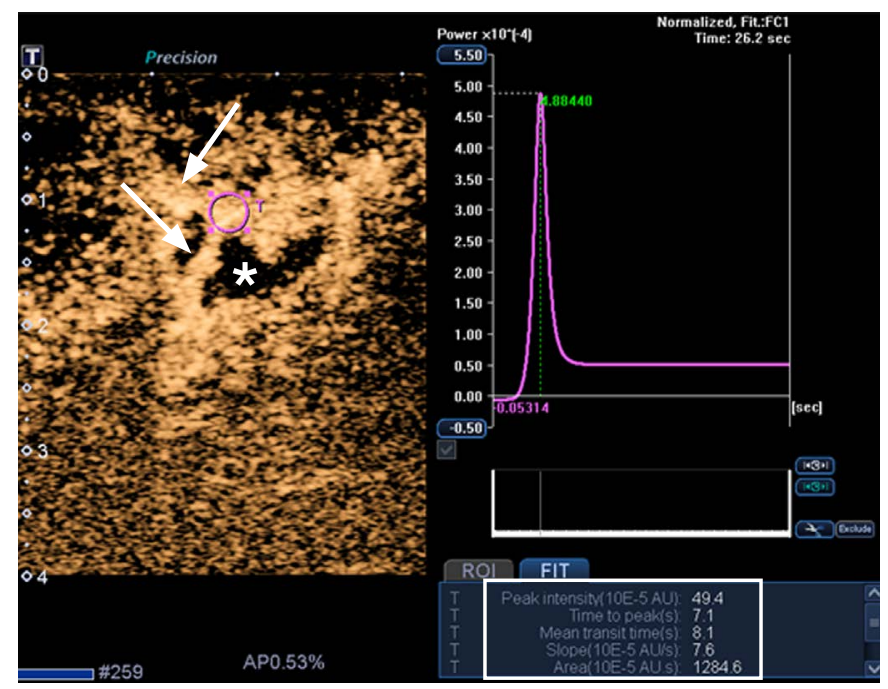

C

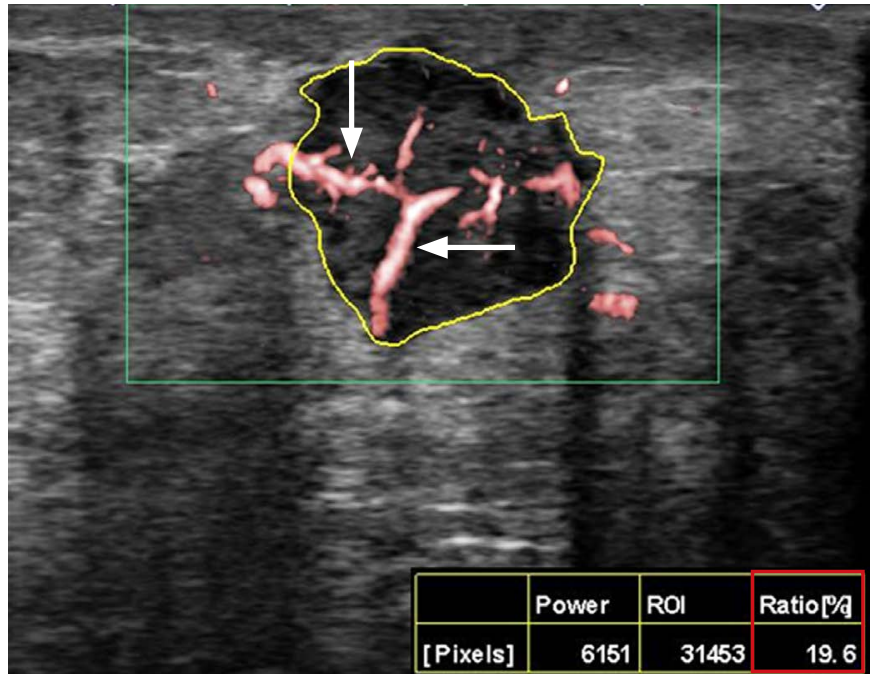

B

Fig. 7. A 74-year-old woman with invasive ductal carcinoma.

A. Gray-scale ultrasonography shows an irregular angular hypoechoic mass in the right breast, assessed as Breast Imaging Reporting and Data System category 4C. B. Superb microvascular imaging shows penetrating (arrows) and branching vessels within the mass. When the region of interest (ROI) is set as the entire target lesion (yellow line), a vascular index of the mass is $19.6 \%$ (red box). C. Contrast-enhanced ultrasonography shows hyperenhancement, penetrating vessels (arrows), and perfusion defect (asterisk) of the lesion. When the ROI (pink circle) is set in the area of the strongest enhancement for time-intensity curve analysis, the mass shows fast and strong enhancement (higher peak intensity, slope, and area under the curve) (white box). 
steps: (1) injection of $3.6 \mathrm{~mL}$ of the contrast agent and continuous scanning for 2 minutes for subsequent time-intensity curve analysis, and (2) injection of another $1.2 \mathrm{~mL}$ of contrast agent after a waiting period of 16 minutes from the first injection for microflow imaging. The built-in software was used for time-intensity curve analysis [29]. An ROI was placed selectively in the area of the strongest enhancement, and the following quantitative parameters were automatically calculated: peak intensity, the maximum intensity of the time-intensity curve; time to peak (TTP), the time needed to reach peak intensity from the time the first microbubble reached the lesion; the mean transit time, the time for which intensity is higher than the mean value; slope, the maximum wash-in velocity; and area under the time-intensity curve, proportional to the total volume of blood in the ROI and the sum of area wash-in and area wash-

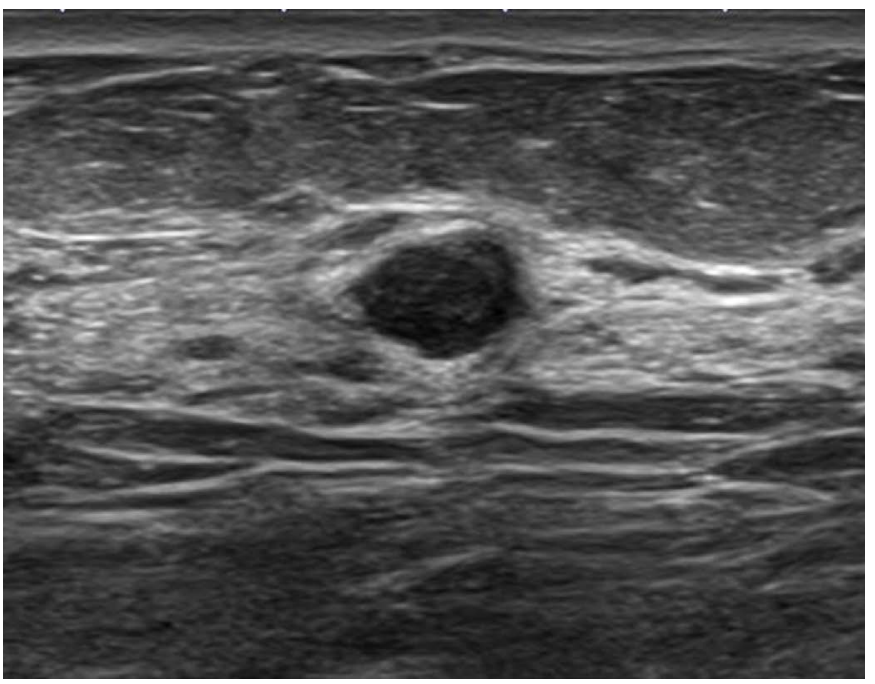

A

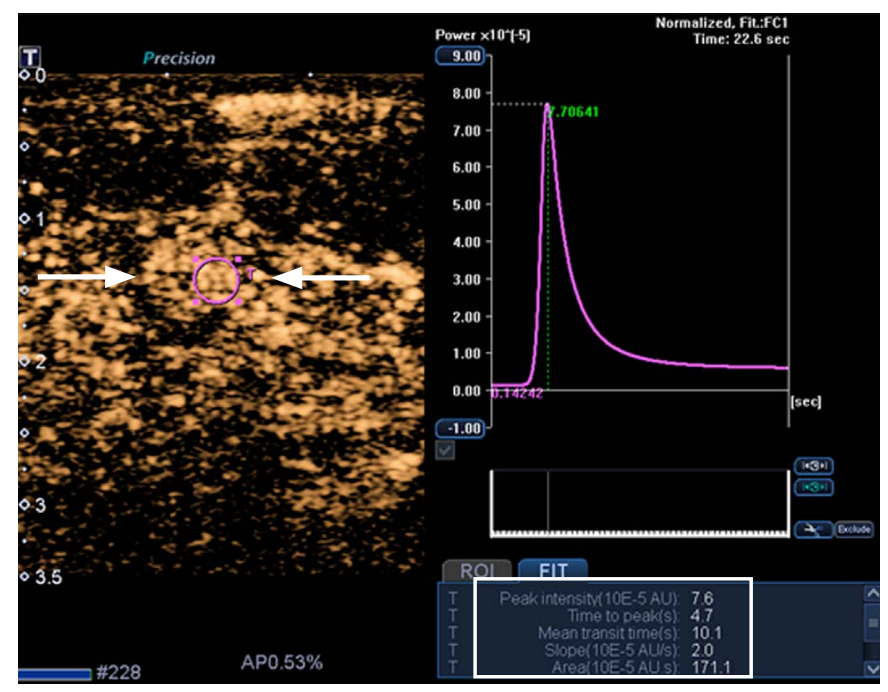

C out. Fig. 6 demonstrates the time-intensity curve of CEUS and its parameters.

\section{Utility of CEUS in Breast Tumor Evaluation}

Many studies have investigated the CEUS features associated with malignant breast tumors and the contribution of CEUS to diagnostic performance, although variation was present in the US equipment, the contrast agent used and its dose, the quantitative analysis software, and the classifications used for image interpretation $[8,21]$. Common signs suggestive of malignancy included heterogeneous or peripheral enhancement, centripetal enhancement order, penetrating vessel and perfusion defect, early intense wash-in, and fast wash-out $[20,21,23-25]$. Several studies have explained these enhancement characteristics in relation to the histopathologic

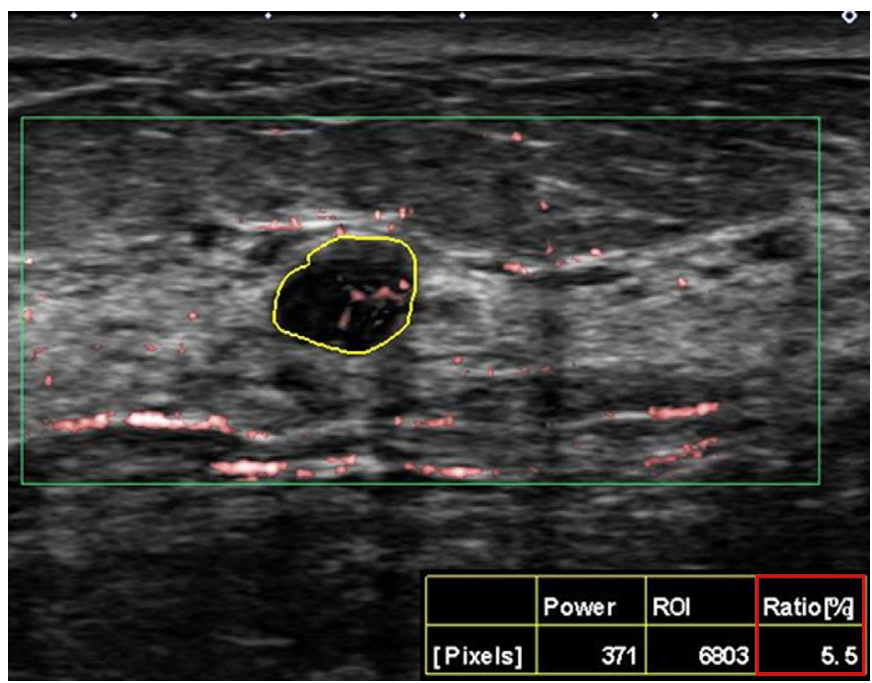

B

Fig. 8. A 36-year-old woman with fibroadenoma.

A. Gray-scale ultrasonography shows an oval microlobulated hypoechoic mass in the left breast, assessed as Breast Imaging Reporting and Data System category 4A. B. Superb microvascular imaging shows linear or dot like vessels within the mass. When the region of interest (ROI) is set as the entire target lesion (yellow line), a vascular index of the mass is $5.5 \%$ (red box). C. Contrastenhanced ultrasonography shows isoenhancement of the lesion (arrows). When the ROI (pink circle) is set in the area of the strongest enhancement for time-intensity curve analysis, the mass shows relatively slow and mild enhancement (lower peak intensity, slope, and area under the curve) (white box). 
features of breast cancer. Liu et al. [30] reported that peripheral enhancement was associated with hypercellularity or adenosis at the periphery and hypocellularity, fibrosis, or necrosis in the center, whereas heterogeneous enhancement was associated with tumor cell cords or clusters in a variable amount of desmoplastic stroma. Wan et al. suggested that early intense wash-in (shorter TTP or higher ascending slope) and fast wash-out were related to highvelocity flow through arteriovenous shunts inside malignant lesions $[20,31]$.

Most studies reported that the addition of CEUS to gray-scale US improved diagnostic performance compared with gray-scale US alone, with sensitivity ranging from $64 \%$ to $100 \%$ and specificity ranging from $38 \%$ to $97 \%$ [20-22,24,26-28,32,33].

In addition, Du et al. [23] reported that the diagnostic performance of a combination of gray-scale US and CEUS was equivalent to that of MRI (AUC, 0.94 vs. 0.91 ; accuracy, 90.2\% vs. $91.8 \%$ ). Ricci et al. [26] also reported equivalent diagnostic performance between CEUS and MRI (sensitivity, $100 \%$ vs. $100 \%$; specificity, $87.5 \%$ vs. $87.5 \%$; accuracy, $94 \%$ vs. $94 \%$ ).

Recently, a clinical study comparing the diagnostic performance of SMI and CEUS in discriminating between malignant and benign breast tumors was published. Xiao et al. [34] evaluated 132 breast tumors with SMI and CEUS and classified the microvascular architecture of breast lesions into five patterns. They concluded that the root hair-like and crab claw-like patterns were associated with breast cancer, and the diagnostic performance of SMI was not different from that of CEUS when using these patterns as diagnostic criteria (sensitivity, $77.6 \%$ vs. $89.6 \%$; specificity, $90.5 \%$ vs. $87.8 \%$; accuracy, $84.8 \%$ vs. $88.6 \%$; AUC, 0.865 vs. 0.791 ) [34].

Figs. 7 and 8 demonstrate the findings of SMI and CEUS for malignant and benign breast tumors.

\section{Potential and Limits of CEUS}

The diagnostic performance of CEUS in discriminating breast cancer from benign tumors has greatly improved due to the development of high-end US systems and the introduction of second-generation US contrast agents. The development of dedicated software also allows the more objective quantitative analysis of lesion vascularity. However, there are no standardized criteria for differentiating between malignant and benign tumors because different US equipment, contrast agents, and quantitative analysis software are currently used, and there is no general consensus about the methods of imaging acquisition and interpretation, such as the dose of contrast agent, the ROI setting for time-intensity curve analysis, and imaging parameters. In addition, the need for intravenous contrast injection and time-consuming post-imaging analysis might explain why CEUS is not routinely used in clinical practice [21].

\section{Summary}

SMI and CEUS can be useful adjunctive US techniques for evaluating breast tumors by assessing the microvasculature. Both quantitative and qualitative analysis of microvessels within breast tumors can provide valuable information for discriminating breast malignancies from benign tumors. However, wider application and further largescale investigations are needed to standardize the methods of image acquisition and interpretation of both US techniques and to achieve consensus about clinically practical diagnostic criteria for diagnosing breast cancer.

Although there have been only a few studies to date, the diagnostic performance of SMI seems to be equivalent to that of CEUS. In consideration of the advantages of SMI, such as not needing contrast agent injection or a time-consuming post-imaging analysis, SMI might be a useful alternative vascular imaging technique to CEUS.

ORCID: Ah Young Park: http://orcid.org/0000-0002-4747-9480; Bo Kyoung Seo: http://orcid.org/0000-0002-9512-5361

\section{Conflict of Interest}

No potential conflict of interest relevant to this article was reported.

\section{Acknowledgments}

We thank Hannah Kim and Sarah Kwon of the ultrasound division of Toshiba Medical Systems Korea Co. Ltd. for their assistance with ultrasound physics.

\section{References}

1. Yadav L, Puri N, Rastogi V, Satpute P, Sharma V. Tumour angiogenesis and angiogenic inhibitors: a review. I Clin Diagn Res 2015;9:XE01-XE05.

2. Folkman J. Angiogenesis and breast cancer. J Clin Oncol 1994;12:441-443.

3. Banerjee $S$, Dowsett M, Ashworth A, Martin LA. Mechanisms of disease: angiogenesis and the management of breast cancer. Nat Clin Pract Oncol 2007;4:536-550.

4. Schneider BP, Miller KD. Angiogenesis of breast cancer. J Clin Oncol 2005;23:1782-1790.

5. Lee SW, Choi HY, Baek SY, Lim SM. Role of color and power Doppler imaging in differentiating between malignant and benign solid breast masses. J Clin Ultrasound 2002;30:459-464.

6. Kook SH, Park HW, Lee YR, Lee YU, Pae WK, Park YL. Evaluation of solid breast lesions with power Doppler sonography. J Clin Ultrasound 1999;27:231-237.

7. Giuseppetti GM, Baldassarre S, Marconi E. Color Doppler 
sonography. Eur J Radiol 1998;27 Suppl 2:S254-S258.

8. Schroeder RJ, Bostanjoglo M, Rademaker J, Maeurer J, Felix R. Role of power Doppler techniques and ultrasound contrast enhancement in the differential diagnosis of focal breast lesions. Eur Radiol 2003;13:68-79.

9. Park AY, Seo BK, Cha SH, Yeom SK, Lee SW, Chung HH. An innovative ultrasound technique for evaluation of tumor vascularity in breast cancers: superb micro-vascular imaging. J Breast Cancer 2016;19:210-213.

10. Hata J. Seeing the unseen new techniques in vascular imaging: superb micro-vascular imaging. Otawara: Toshiba Medical Systems Corporation, 2014.

11 Kruskal JB, Newman PA, Sammons LG, Kane RA. Optimizing Doppler and color flow US: application to hepatic sonography. Radiographics 2004;24:657-675.

12. Ma Y, Li G, Li J, Ren WD. The diagnostic value of superb microvascular imaging (SMI) in detecting blood flow signals of breast lesions: a preliminary study comparing SMI to color Doppler flow imaging. Medicine (Baltimore) 2015;94:e1502.

13. Yongfeng Z, Ping Z, Wengang L, Yang S, Shuangming T. Application of a novel microvascular imaging technique in breast lesion evaluation. Ultrasound Med Biol 2016;42:2097-2105.

14. Zhan J, Diao XH, Jin JM, Chen L, Chen Y. Superb microvascular imaging: a new vascular detecting ultrasonographic technique for avascular breast masses: a preliminary study. Eur J Radiol 2016:85:915-921.

15. Ma Y, Li J, Ren W, Deng L. Correlation between superb microvascular imaging and pathological microvessel density in breast tumors. Xiangya Med 2016;1:30.

16. Adler DD, Carson PL, Rubin JM, Quinn-Reid D. Doppler ultrasound color flow imaging in the study of breast cancer: preliminary findings. Ultrasound Med Biol 1990;16:553-559.

17. Greis C. Technology overview: SonoVue (Bracco, Milan). Eur Radiol 2004;14 Suppl 8:P11-P15.

18. Chung YE, Kim KW. Contrast-enhanced ultrasonography: advance and current status in abdominal imaging. Ultrasonography 2015;34:3-18.

19. Claudon M, Dietrich CF, Choi BI, Cosgrove DO, Kudo M, Nolsoe CP, et al. Guidelines and good clinical practice recommendations for contrast enhanced ultrasound (CEUS) in the liver: update 2012: a WFUMB-EFSUMB initiative in cooperation with representatives of AFSUMB, AIUM, ASUM, FLAUS and ICUS. Ultrasound Med Biol 2013;39:187-210.

20. Wan C, Du J, Fang H, Li F, Wang L. Evaluation of breast lesions by contrast enhanced ultrasound: qualitative and quantitative analysis. Eur J Radiol 2012;81:e444-e450.

21. Drudi FM, Cantisani V, Gnecchi M, Malpassini F, Di Leo N, de Felice C. Contrast-enhanced ultrasound examination of the breast: a literature review. Ultraschall Med 2012;33:E1-E7.
22. Liu H, Jiang YX, Liu JB, Zhu QL, Sun Q. Evaluation of breast lesions with contrast-enhanced ultrasound using the microvascular imaging technique: initial observations. Breast 2008;17:532-539.

23. Du J, Wang L, Wan CF, Hua J, Fang H, Chen J, et al. Differentiating benign from malignant solid breast lesions: combined utility of conventional ultrasound and contrast-enhanced ultrasound in comparison with magnetic resonance imaging. Eur J Radiol 2012;81:3890-3899.

24. Zhao H, Xu R, Ouyang Q, Chen L, Dong B, Huihua Y. Contrastenhanced ultrasound is helpful in the differentiation of malignant and benign breast lesions. Eur J Radiol 2010;73:288-293.

25. Cao XL, Bao W, Zhu SG, Wang LH, Sun MH, Wang L, et al. Contrastenhanced ultrasound characteristics of breast cancer: correlation with prognostic factors. Ultrasound Med Biol 2014;40:11-17.

26. Ricci P, Cantisani V, Ballesio L, Pagliara E, Sallusti E, Drudi FM, et al. Benign and malignant breast lesions: efficacy of real time contrastenhanced ultrasound vs. magnetic resonance imaging. Ultraschall Med 2007;28:57-62.

27. Sorelli PG, Cosgrove DO, Svensson WE, Zaman N, Satchithananda K, Barrett NK, et al. Can contrast-enhanced sonography distinguish benign from malignant breast masses? J Clin Ultrasound 2010;38:177-181.

28. Caproni N, Marchisio F, Pecchi A, Canossi B, Battista R, D'Alimonte $P$, et al. Contrast-enhanced ultrasound in the characterisation of breast masses: utility of quantitative analysis in comparison with MRI. Eur Radiol 2010;20:1384-1395.

29. Lassau N, Chami L, Benatsou B, Peronneau P, Roche A. Dynamic contrast-enhanced ultrasonography (DCE-US) with quantification of tumor perfusion: a new diagnostic tool to evaluate the early effects of antiangiogenic treatment. Eur Radiol 2007;17 Suppl 6:F89-F98.

30. Liu H, Jiang YX, Liu JB, Zhu QL, Sun Q, Chang XY. Contrastenhanced breast ultrasonography: imaging features with histopathologic correlation. J Ultrasound Med 2009;28:911-920.

31. Kedar RP, Cosgrove D, McCready VR, Bamber JC, Carter ER. Microbubble contrast agent for color Doppler US: effect on breast masses: work in progress. Radiology 1996;198:679-686.

32. Clevert $D$, Jung EM, Jungius KP, Ertan K, Kubale R. Value of tissue harmonic imaging (THI) and contrast harmonic imaging (CHI) in detection and characterisation of breast tumours. Eur Radiol 2007; 17:1-10.

33. Miyamoto $Y$, Ito $T$, Takada E, Omoto $K$, Hirai T, Moriyasu F. Efficacy of sonazoid (perflubutane) for contrast-enhanced ultrasound in the differentiation of focal breast lesions: phase 3 multicenter clinical trial. AJR Am J Roentgenol 2014;202:W400-W407.

34. Xiao XY, Chen X, Guan XF, Wu H, Qin W, Luo BM. Superb microvascular imaging in diagnosis of breast lesions: a comparative study with contrast-enhanced ultrasonographic microvascular imaging. Br J Radiol 2016;89:20160546. 